\title{
Phosphodiesterase 3A rs7134375 single nucleotide polymorphism and serum lipid levels
}

\author{
WEI WANG, RUI-XING YIN, DONG-FENG WU, LYNN HTET HTET AUNG, PING HUANG, \\ XIAO-NA ZENG, KE-KE HUANG, QUAN-ZHEN LIN, JIAN WU and TAO GUO \\ Department of Cardiology, Institute of Cardiovascular Diseases, The First Affiliated Hospital, \\ Guangxi Medical University, Nanning, Guangxi 530021, P.R. China
}

Received August 15, 2013; Accepted February 14, 2014

DOI: $10.3892 / \mathrm{mmr} .2014 .2007$

\begin{abstract}
The association between the phosphodiesterase 3A (PDE3A) rs7134375 single nucleotide polymorphism (SNP) and serum lipid levels are not well understood in the general population. The present study was performed in order to detect the association between the rs7134375 SNP and serum lipid levels in the Guangxi Mulao and Han populations. The genotypes of the PDE3A rs7134375 SNP in 761 subjects of the Mulao population and 774 subjects of the Han Chinese population were determined by polymerase chain reaction and restriction fragment length polymorphism combined with gel electrophoresis, and then confirmed by direct sequencing. It was observed that serum low-density lipoprotein cholesterol and apolipoprotein B levels were higher in the Mulao population than in the Han population $(\mathrm{P}<0.05$ for each). The frequencies of the $\mathrm{C}$ and $\mathrm{A}$ alleles were 72.14 and $27.86 \%$ in the Mulao population, and 78.55 and $21.45 \%$ in the Han population $(\mathrm{P}<0.01)$, respectively. The frequencies of the CC, CA and AA genotypes were 52.04, 40.21 and $7.75 \%$ in the Mulao population, and $61.50,34.11$ and $4.39 \%$ in the Han population $(\mathrm{P}<0.01)$, respectively. The frequencies of the $\mathrm{C}$ and $\mathrm{A}$ alleles were 74.89 and $25.11 \%$ in Mulao females, and 68.08 and $31.92 \%$ in Mulao males $(\mathrm{P}<0.01)$, respectively. The serum triglyceride (TG) levels were different among the genotypes in the Mulao population; however, not in the Han population $(\mathrm{P}<0.01)$, and the $\mathrm{A}$ allele carriers exhibited lower TG levels than the A allele noncarriers. The serum lipid parameters were also correlated with several environmental factors in the two ethnic groups $(\mathrm{P}<0.05-0.001)$. It was concluded that the genotypic and allelic frequencies of the rs7134375 SNP are different between the Mulao and
\end{abstract}

Correspondence to: Professor Rui-Xing Yin, Department of Cardiology, Institute of Cardiovascular Diseases, The First Affiliated Hospital, Guangxi Medical University, 22 Shuangyong Road, Nanning, Guangxi 530021, P.R. China

E-mail: yinruixing@163.com

Key words: phosphodiesterase 3A gene, single nucleotide polymorphism, lipids, apolipoproteins
Han populations. In addition, the PDE3A rs7134375 SNP is associated with serum TG levels in the Mulao population, however, not in the Han population.

\section{Introduction}

Coronary heart disease (CHD) is the leading cause of mortality and disability worldwide (1). Consistent and compelling evidence has demonstrated the association between dyslipidemia and CHD (2-5). Dyslipidemia, particularly increased serum low-density lipoprotein cholesterol (LDL-C) and triglyceride (TG) levels, is a well-described risk factor for atherosclerosis and CHD (6). It is well established that dyslipidemia is a complex trait caused by multiple environmental and genetic factors and their interactions (7-10). Family studies suggest that in numerous populations, approximately half of the variation in serum lipid profiles is genetically determined (11). Previous genome-wide association studies (GWAS) in different populations have identified $>95$ loci associated with serum lipid levels (12-22). Common variants at these loci together explain $<10 \%$ of variation in each lipid trait. Rare variants with large individual effects may also contribute to the heritability of lipid traits (23). Phosphodiesterase 3A (PDE3A), a gene located on chromosome $12 \mathrm{p} 12$, is a member of the PDE family, and is located in an intron of the gene. PDEs selectively catalyze the hydrolysis of $3^{\prime}$ cyclic phosphate bonds in adenosine and/or guanine 3', 5' cyclic monophosphate (cAMP and/or cGMP). They regulate the cellular levels, localization and duration of action of these second messengers by controlling the rate of their degradation. PDE3A has a high affinity for cAMP and cGMP, and demonstrates competitive inhibition of the cAMP hydrolytic activity of cGMP. It is important in regulating intracellular levels of cAMP and cGMP (24-28). The rs7134375 single nucleotide polymorphism (SNP) with an A/C variation in $P D E 3 A$ has been associated with high-density lipoprotein cholesterol (HDL-C) $(12,29)$; however, it remains to be elucidated whether other serum lipid parameters have a connection with it.

China is a multi-ethnic country with 56 ethnic groups. The Han nationality is the largest ethnic group and the Mulao nationality is one of the 55 ethnic minorities with a population of 207,352 according to the fifth national census statistics 
of China in 2000 (30). The majority of this population have been living in the Luocheng Mulao Autonomous County (Guangxi Zhuang Autonomous Region, China). The history of this minority can be traced back to the Jin Dynasty (AD 265-420). A previous study demonstrated that the genetic association between the Mulao nationality and other minorities in Guangxi was significantly closer than that between the Mulao and Han or Uighur nationality (31). To the best of our knowledge, however, the association between the rs7134375 SNP in PDE3A and serum lipid levels has not been previously reported in this population. Therefore, the present study was performed to evaluate the association between the rs 7134375 SNP and several environmental factors with serum lipid levels in the Guangxi Mulao and Han populations.

\section{Materials and methods}

Study population. A total of 761 Mulao subjects who reside in the Luocheng Mulao Autonomous County and 774 Han subjects who reside in the same villages were included in the present study. The subjects of the Mulao population consisted of 307 (40.34\%) males and 454 (59.66\%) females, ranging between 16 and 93 years old, with a mean age of $51.86 \pm 14.82$ years. The subjects of the Han population consisted of 286 (36.95\%) males and $488(63.05 \%)$ females, aged 16-92 years, with a mean age of 52.05 \pm 14.95 years. All the subjects were randomly selected from our previous stratified randomized samples $(32,33)$. All the study subjects were healthy rural agricultural workers and had no evidence of diseases associated with atherosclerosis, CHD or diabetes. The participants were not taking medications known to affect serum lipid levels (for example lipid-lowering drugs, including statins or fibrates, beta-blockers, diuretics, contraceptives or hormones). The present study was approved by the Ethics Committee of the First Affiliated Hospital, Guangxi Medical University (Nanning, China). Informed consent was obtained from all the subjects following receiving a full explanation of the present study.

Epidemiological survey. The survey was performed using internationally standardized methods (34). Information concerning demographics, socioeconomic status and lifestyle factors was collected with standardized questionnaires. The information collected regarding alcohol intake, included the number of Liangs ( $\sim 50 \mathrm{~g})$ of rice wine, corn wine, rum, beer or liquor consumed during the preceding 12 months. Alcohol consumption was categorized into two groups depending on the grams of alcohol intake per day: $\leq 25$ and $>25$. Smoking status was also categorized into groups depending on the number of cigarettes per day: $\leq 20$ and $>20$. Height, weight and waist circumference were manually measured under the supervision of two individuals. Sitting blood pressure was measured using a mercury sphygmomanometer (Jiangsu Yuwell Medical Equipment and Supply Co., Ltd., Danyang, China). during three separate intervals after a $5 \mathrm{~min}$ rest period, and the average of the three measurements was used taken as the blood pressure. Body mass index (BMI) was calculated as the weight in $\mathrm{kg}$ divided by the square of height in meters $\left(\mathrm{kg} / \mathrm{m}^{2}\right)$.

Biochemical analysis. Venous blood samples were obtained from all subjects following $\geq 12 \mathrm{~h}$ of fasting. The levels of serum total cholesterol (TC), TG, HDL-C and LDL-C in samples were determined by enzymatic methods with commercially available kits (Tcho-1 and TG-LH; Randox Laboratories Ltd., Crumlin, UK; Cholestest N HDL and Cholestest LDL; Daiichi Pure Chemicals Co., Ltd., Tokyo, Japan, respectively). Serum apolipoprotein (Apo) AI and ApoB levels were detected by the immunoturbidimetric immunoassay using a commercial kit (APO CAL; LP3023; Randox Laboratories Ltd.) (32,33).

DNA amplification and genotyping. Genomic DNA was extracted from peripheral blood leukocytes using the phenol-chloroform method (32-35). The extracted DNA was stored at $4^{\circ} \mathrm{C}$ for future analysis. Genotyping of the rs7134375 SNP was performed using a pair of primers: Forward: 5'-TGGGAATCGTTCTTGTTT-3' and reverse: 5'-GAAAGCCTAAGAGTAATTCATG-3' (Sangon, Shanghai, China). Each $20 \mu \mathrm{l}$ PCR reaction mixture consisted of $1 \mu \mathrm{l}$ genomic DNA, $0.8 \mu \mathrm{l}$ of each primer (10 pmol/1), $10 \mu \mathrm{l}$ of 2X Taq PCR Mastermix (20 mM Tris-HCl, pH 8.3; $100 \mathrm{mM}$ $\mathrm{KCl}, 3 \mathrm{mM} \mathrm{MgCl}{ }_{2}, 0.1$ units Taq Polymerase/ $\mu$ l and $500 \mu \mathrm{M}$ dNTP each) and $8 \mu \mathrm{lddH_{2 }} \mathrm{O}$ (DNase/RNase-free). The reaction mixture was subjected to denaturation at $95^{\circ} \mathrm{C}$ for $5 \mathrm{~min}$, followed by 33 cycles at $95^{\circ} \mathrm{C}$ for $45 \mathrm{sec}, 51^{\circ} \mathrm{C}$ for $30 \mathrm{sec}, 72^{\circ} \mathrm{C}$ for $50 \mathrm{sec}$ and a final extension at $72^{\circ} \mathrm{C}$ for $10 \mathrm{~min}$. Then, the amplification products $(5 \mathrm{ml})$ were digested using 5 units MspI restriction enzyme (Fermentas Co., Burlington, ON, Canada) at $37^{\circ} \mathrm{C}$ overnight. Following restriction enzyme digestion of the amplified DNA, the genotypes were identified by electrophoresis on $2 \%$ agarose gels and visualized with ethidium-bromide staining ultraviolet illumination. The genotypes were scored by an experienced reader blinded to epidemiological data and serum lipid levels. Six samples (CC, CA and AA genotypes in two, respectively) detected by PCR-restriction fragment length polymorphism (RFLP) were also confirmed by direct sequencing. The PCR products were purified by low melting point gel electrophoresis and phenol extraction, and then the DNA sequences were analyzed by Shanghai Sangon Biological Engineering Technology \& Services Co., Ltd. (Shanghai, China).

Diagnostic criteria. The normal values of serum TC, TG, HDL-C, LDL-C, ApoAI and ApoB levels, and the ratio of ApoAI to ApoB in our Clinical Science Experiment Center were $3.10-5.17,0.56-1.70,0.91-1.81,2.70-3.20 \mathrm{mmol} / 1$, 1.00-1.78, 0.63-1.14 g/l and 1.00-2.50, respectively $(32,35)$. Hypertension was diagnosed according to the criteria of the 1999 World Health Organization-International Society of Hypertension Guidelines for the Management of Hypertension (36). Normal weight, overweight and obese were defined as a BMI <24, 24-28 and $>28 \mathrm{~kg} / \mathrm{m}^{2}$, respectively (37).

Statistical analysis. All statistical analyses were performed using the statistical software package SPSS 13.0 (SPSS, Inc., Chicago, IL, USA). Qualitative variables are expressed as the raw count and percentage. The mean \pm standard deviation was used for the presentation of quantitative variables. Genotypic and allelic frequencies were calculated by direct counting and the standard goodness-of-fit test was used to assess the Hardy-Weinberg equilibrium. A $\chi^{2}$ test was used 
Table I. Comparison of general characteristics and serum lipid levels between Mulao and Han populations.

\begin{tabular}{|c|c|c|c|c|}
\hline Parameter & Han & Mulao & $\mathrm{t}\left(\chi^{2}\right)$ & P-value \\
\hline Number & 774 & 761 & & \\
\hline Male/female & $286 / 488$ & $307 / 454$ & 1.861 & 0.173 \\
\hline Age (years) & $52.05 \pm 14.95$ & $51.86 \pm 14.82$ & 0.253 & 0.803 \\
\hline Height (cm) & $154.24 \pm 7.83$ & $155.84 \pm 7.89$ & 1.489 & 0.137 \\
\hline Weight (kg) & $53.16 \pm 8.73$ & $52.49 \pm 9.18$ & 1.466 & 0.143 \\
\hline Body mass index $\left(\mathrm{kg} / \mathrm{m}^{2}\right)$ & $22.10 \pm 3.35$ & $21.83 \pm 3.06$ & 1.645 & 0.100 \\
\hline Waist circumference $(\mathrm{cm})$ & $75.20 \pm 7.84$ & $74.86 \pm 7.47$ & 0.403 & 0.416 \\
\hline \multicolumn{5}{|l|}{ Cigarette smoking (n \%) } \\
\hline Nonsmoker & $584(75.45)$ & $594(78.06)$ & & \\
\hline$\leq 20$ cigarettes/day & $168(21.71)$ & $142(18.66)$ & 2.347 & 0.309 \\
\hline$>20$ cigarettes/day & $22(2.84)$ & $25(3.28)$ & & \\
\hline \multicolumn{5}{|l|}{ Alcohol consumption [n (\%)] } \\
\hline Nondrinker & $624(80.62)$ & $602(79.11)$ & & \\
\hline$\leq 25 \mathrm{~g} /$ day & $72(9.30)$ & $60(7.88)$ & 3.867 & 0.145 \\
\hline$>25 \mathrm{~g} /$ day & $78(10.08)$ & $99(13.01)$ & & \\
\hline Systolic blood pressure (mmHg) & $129.11 \pm 19.26$ & $128.86 \pm 21.40$ & 0.246 & 0.806 \\
\hline Diastolic blood pressure (mmHg) & $81.98 \pm 11.19$ & $81.040 \pm 11.25$ & 1.637 & 0.102 \\
\hline Pulse pressure (mmHg) & $47.13 \pm 14.42$ & $47.82 \pm 16.17$ & 0.872 & 0.383 \\
\hline Glucose (mmol/l) & $6.28 \pm 1.79$ & $6.19 \pm 1.67$ & 0.457 & 0.511 \\
\hline Total cholesterol (mmol/l) & $4.96 \pm 1.05$ & $5.08 \pm 1.24$ & 1.883 & 0.060 \\
\hline Triglycerides (mmol/l) & $1.39 \pm 1.35$ & $1.36 \pm 0.94$ & 0.499 & 0.618 \\
\hline HDL-C (mmol/l) & $1.73 \pm 0.53$ & $1.77 \pm 0.45$ & 1.583 & 0.114 \\
\hline LDL-C (mmol/l) & $2.87 \pm 0.87$ & $2.98 \pm 0.91$ & -2.565 & 0.010 \\
\hline ApoAI (g/l) & $1.33 \pm 0.26$ & $1.33 \pm 0.42$ & 0.183 & 0.855 \\
\hline ApoB (g/l) & $0.85 \pm 0.20$ & $0.98 \pm 0.54$ & -6.394 & 0.000 \\
\hline ApoAI/ApoB & $1.65 \pm 0.50$ & $1.59 \pm 0.94$ & 1.508 & 0.130 \\
\hline
\end{tabular}

HDL-C, high-density lipoprotein cholesterol; LDL-C, low-density lipoprotein cholesterol; ApoAI, apolipoprotein AI; ApoB, apolipoprotein B.

to evaluate the difference in genotype distribution and gender ratio between the groups. The difference in general characteristics between the Mulao and Han populations was assessed using Student's unpaired t-test. The association between the genotypes and serum lipid parameters was examined using analysis of covariance. Gender, age, BMI, blood pressure, alcohol consumption and cigarette smoking were adjusted for the statistical analysis. In order to evaluate the correlation between serum lipid parameters and several environmental factors, multiple linear regression analysis with stepwise modeling was also performed. Two-tailed $\mathrm{P}<0.05$ was considered to indicate a statistically significant difference.

\section{Results}

General characteristics and serum lipid levels. Table I shows the general characteristics and serum lipid parameters of the study populations. As compared with the Han population, the Mulao population had higher serum LDL-C and ApoB levels $(\mathrm{P}<0.05$ for each). No significant differences in the age, height, weight, BMI, waist circumference, blood pressure, serum glucose, TC, TG, HDL-C, ApoAI, ApoB, the ratio of ApoAI

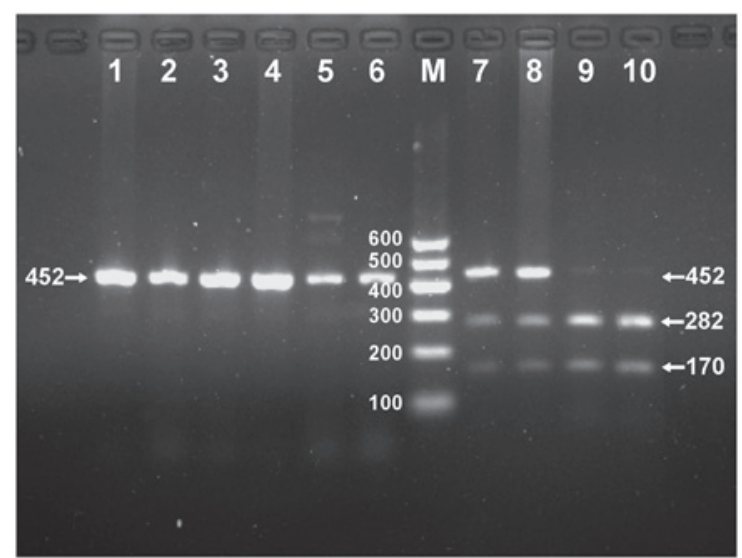

Figure 1. Genotyping of the rs7134375 single nucleotide polymorphism in the phosphodiesterase 3A gene. Lane M, 100 bp marker ladder; lanes 1-4, PCR products (452 bp); lanes 5 and 6, AA genotype (452 bp); lanes 7 and 8 , AC genotype (452, 282 and $170 \mathrm{bp})$, and lanes 9 and 10, CC genotype (282 and $170 \mathrm{bp})$.

to ApoB, the percentages of subjects who smoked cigarettes and the ratio of males to females were identified between the two ethnic groups ( $\mathrm{P}>0.05$ for all). 
Table II. Comparison of the genotypic and allelic frequencies of the rs7134375 single nucleotide polymorphism between the Mulao and Han populations [n (\%)].

\begin{tabular}{|c|c|c|c|c|c|c|}
\hline \multirow[b]{2}{*}{ Group } & \multirow[b]{2}{*}{$\mathrm{n}$} & \multicolumn{3}{|c|}{ Genotype } & \multicolumn{2}{|c|}{ Allele } \\
\hline & & $\mathrm{CC}$ & $\mathrm{CA}$ & AA & $\mathrm{C}$ & A \\
\hline Mulao & 761 & $396(52.04)$ & $306(40.21)$ & $59(7.75)$ & $1098(72.14)$ & $424(27.86)$ \\
\hline Han & 774 & $476(61.50)$ & $264(34.11)$ & 34 (4.39) & $1216(78.55)$ & $332(21.45)$ \\
\hline$\chi^{2}$ & - & 17.046 & & & 16.994 & \\
\hline P-value & - & 0.000 & & & 0.000 & \\
\hline \multicolumn{7}{|l|}{ Mulao } \\
\hline Male & 307 & $148(48.21)$ & $122(39.74)$ & $37(12.05)$ & $418(68.08)$ & $196(31.92)$ \\
\hline Female & 454 & $248(54.63)$ & $184(40.53)$ & $22(4.84)$ & $680(74.89)$ & $228(25.11)$ \\
\hline$\chi^{2}$ & - & 13.746 & & & 8.457 & \\
\hline P-value & - & 0.001 & & & 0.004 & \\
\hline \multicolumn{7}{|l|}{ Han } \\
\hline Male & 355 & $193(54.4)$ & $140(39.4)$ & $22(6.20)$ & $526(74.1)$ & 184 (25.9) \\
\hline Female & 452 & $252(55.8)$ & $162(35.8)$ & $38(8.4)$ & $666(73.7)$ & $238(26.3)$ \\
\hline$\chi^{2}$ & - & 2.062 & & & 0.035 & \\
\hline P-value & - & 0.357 & & & 0.852 & \\
\hline
\end{tabular}
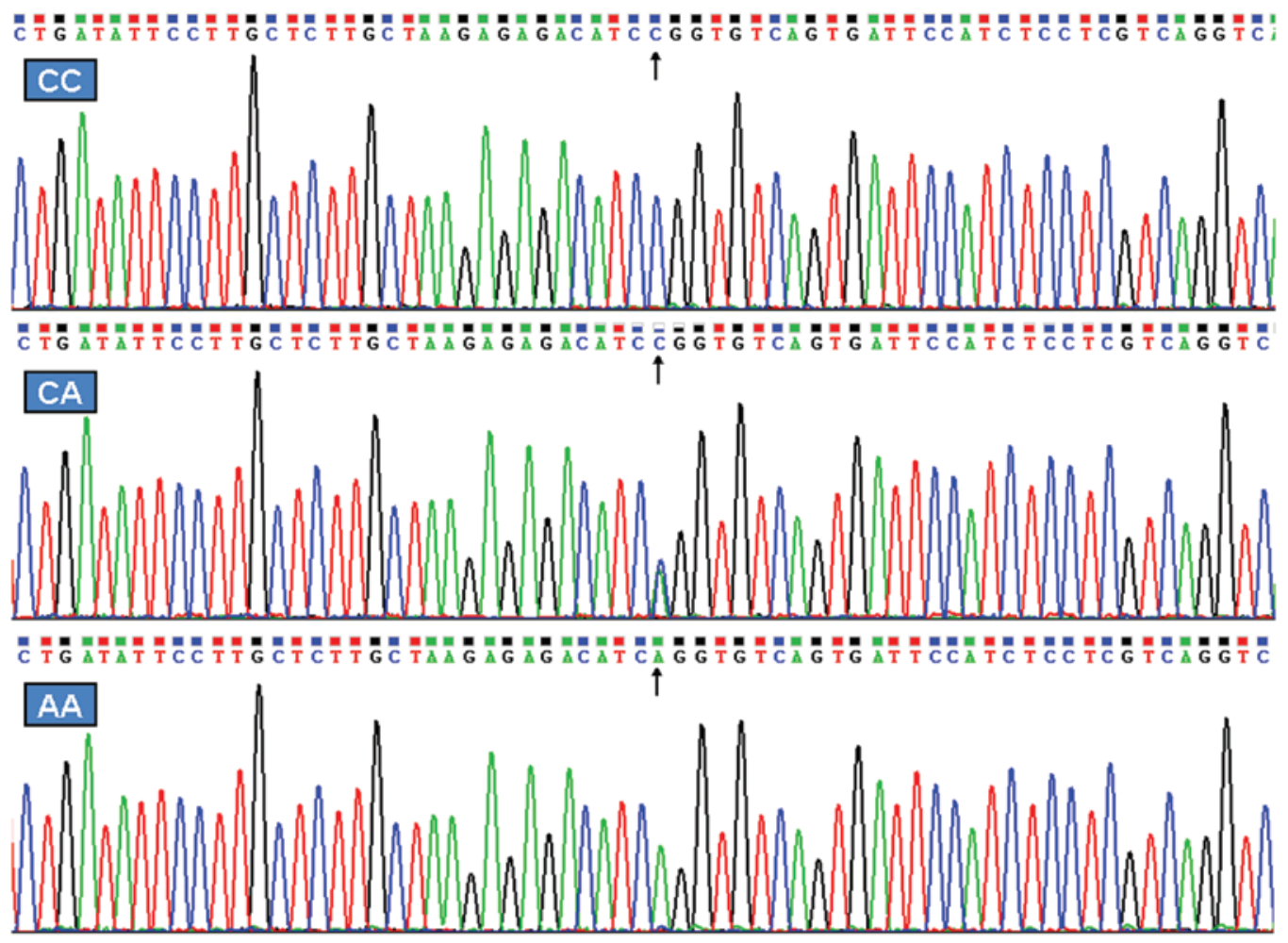

Figure 2. A section of the nucleotide sequence of the rs7134375 single nucleotide polymorphism in the phosphodiesterase $3 \mathrm{~A}$ gene. The CC, CA and AA genotypes are shown, respectively.

Results of electrophoresis, genotyping and sequencing. Following amplification of the genomic DNA of the samples by PCR, the PCR products of 452 bp nucleotide sequences were observed in the samples (lanes 1-4; Fig. 1). The genotypes identified were named according to the presence ( $\mathrm{C}$ allele) or absence (A allele) of the enzyme restriction sites. Thus, the AA genotype is heterozygote for the absence of the site (bands at
452 bp; lanes 5 and 6; Fig. 1), the CA genotype is heterozygote for the absence and presence of the site (bands at 452, 282 and 170 bp; lanes 7 and 8; Fig. 1) and the CC genotype is homozygote for the presence of the site (bands at 282 and $170 \mathrm{bp}$; lanes 9 and 10; Fig. 1). The AA, CA and CC genotypes detected by the PCR-RFLP were also confirmed by sequencing (Fig. 2), respectively. 
Table III. Genotypes of the rs7134375 single nucleotide polymorphism and serum lipid levels in the Mulao and Han populations.

\begin{tabular}{|c|c|c|c|c|c|c|c|c|}
\hline Genotype & $\mathrm{n}$ & $\begin{array}{c}\mathrm{TC} \\
(\mathrm{mmol} / \mathrm{l})\end{array}$ & $\begin{array}{c}\mathrm{TG} \\
(\mathrm{mmol} / \mathrm{l})\end{array}$ & $\begin{array}{l}\text { HDL-C } \\
(\mathrm{mmol} / \mathrm{l})\end{array}$ & $\begin{array}{l}\text { LDL-C } \\
(\mathrm{mmol} / \mathrm{l})\end{array}$ & $\begin{array}{c}\text { ApoAI } \\
(\mathrm{g} / \mathrm{l})\end{array}$ & $\begin{array}{c}\text { ApoB } \\
(\mathrm{g} / \mathrm{l})\end{array}$ & ApoAI/ApoB \\
\hline \multicolumn{9}{|l|}{ Mulao } \\
\hline $\mathrm{CC}$ & 396 & $5.10 \pm 1.34$ & $1.14(0.87)$ & $1.78 \pm 0.48$ & $3.03 \pm 0.91$ & $1.35 \pm 0.42$ & $0.97 \pm 0.51$ & $1.58 \pm 0.68$ \\
\hline $\mathrm{CA}$ & 306 & $5.08 \pm 1.15$ & $1.03(0.66)$ & $1.76 \pm 0.42$ & $2.95 \pm 0.94$ & $1.31 \pm 0.38$ & $0.99 \pm 0.58$ & $1.63 \pm 1.24$ \\
\hline AA & 59 & $4.88 \pm 0.86$ & $1.01(0.97)$ & $1.71 \pm 0.45$ & $2.89 \pm 0.78$ & $1.27 \pm 0.41$ & $0.99 \pm 0.54$ & $1.48 \pm 0.64$ \\
\hline F-value & - & 0.870 & 7.010 & 0.809 & 1.911 & 1.757 & 0.054 & 0.635 \\
\hline P-value & - & 0.420 & 0.030 & 0.445 & 0.149 & 0.173 & 0.947 & 0.530 \\
\hline $\mathrm{CC}$ & 396 & $5.10 \pm 1.34$ & $1.14(0.87)$ & $1.78 \pm 0.48$ & $3.03 \pm 0.91$ & $1.35 \pm 0.42$ & $0.97 \pm 0.51$ & $1.58 \pm 0.68$ \\
\hline $\mathrm{CA} / \mathrm{AA}$ & 365 & $5.05 \pm 1.11$ & $1.02(0.66)$ & $1.75 \pm 0.42$ & $2.94 \pm 0.94$ & $1.30 \pm 0.39$ & $0.99 \pm 0.56$ & $1.61 \pm 1.17$ \\
\hline F-value & - & 0.638 & 2.605 & 0.980 & 1.303 & 1.783 & -0.313 & -0.331 \\
\hline P-value & - & 0.524 & 0.009 & 0.327 & 0.193 & 0.075 & 0.755 & 0.741 \\
\hline \multicolumn{9}{|l|}{ Han } \\
\hline $\mathrm{CC}$ & 476 & $4.97 \pm 1.02$ & $1.03(0.90)$ & $1.71 \pm 0.42$ & $2.84 \pm 0.84$ & $1.34 \pm 0.26$ & $0.85 \pm 0.21$ & $1.65 \pm 0.47$ \\
\hline $\mathrm{CA}$ & 264 & $4.93 \pm 1.09$ & $1.06(0.71)$ & $1.76 \pm 0.69$ & $2.82 \pm 0.92$ & $1.32 \pm 0.27$ & $0.83 \pm 0.21$ & $1.67 \pm 0.55$ \\
\hline AA & 34 & $5.17 \pm 1.10$ & $1.22(0.97)$ & $1.71 \pm 0.46$ & $3.12 \pm 0.80$ & $1.35 \pm 0.23$ & $0.91 \pm 0.13$ & $1.50 \pm 0.30$ \\
\hline F-value & - & 0.797 & 1.372 & 0.613 & 1.686 & 0.676 & 2.352 & 1.751 \\
\hline P-value & - & 0.451 & 0.503 & 0.542 & 0.186 & 0.509 & 0.096 & 0.174 \\
\hline $\mathrm{CC}$ & 476 & $4.97 \pm 1.02$ & $1.03(0.90)$ & $1.71 \pm 0.42$ & $2.84 \pm 0.84$ & $1.34 \pm 0.26$ & $0.85 \pm 0.21$ & $1.65 \pm 0.47$ \\
\hline $\mathrm{CA} / \mathrm{AA}$ & 298 & $4.96 \pm 1.09$ & $1.08(0.73)$ & $1.75 \pm 0.67$ & $2.91 \pm 0.91$ & $1.33 \pm 0.27$ & $0.84 \pm 0.20$ & $1.65 \pm 0.53$ \\
\hline F-value & - & 0.011 & -0.231 & -0.998 & -0.967 & 0.973 & 0.604 & -0.001 \\
\hline P-value & - & 0.915 & 0.817 & 0.318 & 0.334 & 0.331 & 0.546 & 0.999 \\
\hline \multicolumn{9}{|c|}{ Mulao/male } \\
\hline $\mathrm{CC}$ & 148 & $4.98 \pm 1.64$ & $1.36 \pm 0.97$ & $1.78 \pm 0.53$ & $2.82 \pm 0.89$ & $1.34 \pm 0.43$ & $0.99 \pm 0.67$ & $1.65 \pm 0.78$ \\
\hline $\mathrm{CA} / \mathrm{AA}$ & 159 & $4.72 \pm 1.07$ & $1.17 \pm 0.83$ & $1.70 \pm 0.44$ & $2.73 \pm 0.88$ & $1.31 \pm 0.40$ & $0.91 \pm 0.53$ & $1.82 \pm 1.60$ \\
\hline F-value & - & 1.684 & 1.821 & 1.356 & 0.862 & 0.629 & 1.138 & -1.186 \\
\hline P-value & - & 0.093 & 0.070 & 0.176 & 0.389 & 0.530 & 0.256 & 0.237 \\
\hline \multicolumn{9}{|c|}{ Mulao/female } \\
\hline $\mathrm{CC}$ & 248 & $5.17 \pm 1.12$ & $1.47 \pm 0.99$ & $1.78 \pm 0.45$ & $3.15 \pm 0.90$ & $1.36 \pm 0.41$ & $0.96 \pm 0.39$ & $1.54 \pm 0.60$ \\
\hline $\mathrm{CA} / \mathrm{AA}$ & 206 & $5.30 \pm 1.08$ & $1.36 \pm 0.93$ & $1.78 \pm 0.40$ & $3.10 \pm 0.91$ & $1.30 \pm 0.38$ & $1.05 \pm 0.58$ & $1.44 \pm 0.60$ \\
\hline F-value & - & -1.194 & 1.243 & -0.027 & 0.582 & 1.789 & -1.723 & 1.837 \\
\hline P-value & - & 0.233 & 0.214 & 0.978 & 0.561 & 0.074 & 0.085 & 0.067 \\
\hline \multicolumn{9}{|l|}{ Han/male } \\
\hline $\mathrm{CC}$ & 172 & $4.94 \pm 1.00$ & $1.47 \pm 1.10$ & $1.68 \pm 0.41$ & $2.85 \pm 0.85$ & $1.30 \pm 0.25$ & $0.85 \pm 0.20$ & $1.62 \pm 0.47$ \\
\hline $\mathrm{CA} / \mathrm{AA}$ & 114 & $4.99 \pm 1.06$ & $1.37 \pm 1.00$ & $1.70 \pm 0.41$ & $2.93 \pm 0.91$ & $1.29 \pm 0.25$ & $0.85 \pm 0.21$ & $1.62 \pm 0.58$ \\
\hline F-value & - & -0.376 & 0.808 & -0.381 & -0.758 & 0.340 & -0.060 & -0.034 \\
\hline P-value & - & 0.707 & 0.420 & 0.704 & 0.449 & 0.734 & 0.952 & 0.973 \\
\hline \multicolumn{9}{|c|}{ Han/female } \\
\hline $\mathrm{CC}$ & 304 & $4.98 \pm 1.03$ & $1.025(0.760)$ & $1.73 \pm 0.42$ & $2.84 \pm 0.84$ & $1.36 \pm 0.25$ & $0.85 \pm 0.21$ & $1.67 \pm 0.47$ \\
\hline $\mathrm{CA} / \mathrm{AA}$ & 184 & $4.94 \pm 1.12$ & $1.025(0.760)$ & $1.78 \pm 0.79$ & $2.89 \pm 0.90$ & $1.33 \pm 0.28$ & $0.84 \pm 0.20$ & $1.67 \pm 0.51$ \\
\hline F-value & - & 0.413 & -0.371 & -0.972 & -0.626 & 0.896 & 0.803 & -0.014 \\
\hline P-value & - & 0.680 & 0.710 & 0.332 & 0.532 & 0.371 & 0.422 & 0.989 \\
\hline
\end{tabular}

TC, total cholesterol; TG, triglyceride; HDL-C, high-density lipoprotein cholesterol; LDL-C, low-density lipoprotein cholesterol; ApoAI, apolipoprotein AI; ApoB, apolipoprotein B. The value for TG is presented as the median (interquartile range). The difference between the two ethnic groups was determined by the Wilcoxon-Mann-Whitney test.

Genotypic and allelic frequencies. The genotypic and allelic frequencies of the PDE3A rs7134375 SNP are shown in Table II. The frequency of $\mathrm{C}$ and $\mathrm{A}$ alleles was 78.55 and $21.45 \%$ in the Han population, and 72.14 and $27.86 \%$ in the Mulao population, respectively. The frequency of CC, CA and AA genotypes was $61.50,34.11$ and $4.39 \%$ in the Han population, and 52.04, 40.21 and $7.75 \%$ in the Mulao population, respectively. The genotypic and allelic frequencies were different between the Mulao and 
Table IV. Association between serum lipid parameters and relative factors in the Mulao and Han populations.

A, Han and Mulao

\begin{tabular}{|c|c|c|c|c|c|c|}
\hline Lipid parameter & Risk factor & $\begin{array}{c}\text { Unstandardized } \\
\text { Coefficient }\end{array}$ & Std. error & $\begin{array}{l}\text { Standardized } \\
\text { coefficient }\end{array}$ & $\mathrm{t}$ & P-value \\
\hline \multirow[t]{5}{*}{$\mathrm{TC}$} & Age & 0.013 & 0.002 & 0.163 & 6.359 & 0.000 \\
\hline & Waist circumference & 0.016 & 0.004 & 0.113 & 4.379 & 0.000 \\
\hline & Alcohol consumption & 0.003 & 0.001 & 0.100 & 3.985 & 0.000 \\
\hline & Diastolic blood pressure & 0.006 & 0.003 & 0.055 & 2.053 & 0.040 \\
\hline & Ethnic group & -0.112 & 0.057 & -0.049 & -1.977 & 0.048 \\
\hline \multirow[t]{3}{*}{ TG } & Waist circumference & 0.041 & 0.004 & 0.287 & 11.556 & 0.000 \\
\hline & Systolic blood pressure & 0.004 & 0.001 & 0.068 & 2.749 & 0.006 \\
\hline & Alcohol consumption & 0.002 & 0.001 & 0.065 & 2.677 & 0.008 \\
\hline \multirow[t]{3}{*}{ HDL-C } & Waist circumference & -0.014 & 0.002 & -0.234 & -9.375 & 0.000 \\
\hline & Alcohol consumption & 0.002 & 0.000 & 0.098 & 3.949 & 0.000 \\
\hline & Age & 0.001 & 0.001 & 0.065 & 2.628 & 0.009 \\
\hline \multirow[t]{4}{*}{ LDL-C } & Age & 0.011 & 0.001 & 0.183 & 7.426 & 0.000 \\
\hline & Body mass index & 0.032 & 0.009 & 0.117 & 3.433 & 0.001 \\
\hline & Ethnic group & -0.131 & 0.044 & -0.073 & -2.979 & 0.003 \\
\hline & Waist circumference & 0.009 & 0.004 & 0.085 & 2.500 & 0.013 \\
\hline \multirow[t]{2}{*}{ ApoAI } & Alcohol consumption & 0.002 & 0.000 & 0.201 & 8.014 & 0.000 \\
\hline & Waist circumference & -0.004 & 0.001 & -0.100 & -3.976 & 0.000 \\
\hline \multirow[t]{4}{*}{ ApoB } & Waist circumference & 0.010 & 0.001 & 0.207 & 8.150 & 0.000 \\
\hline & Ethnic group & -1.134 & 0.020 & -0.163 & -6.655 & 0.000 \\
\hline & Systolic blood pressure & 0.003 & 0.001 & 0.160 & 4.874 & 0.000 \\
\hline & Diastolic blood pressure & -0.003 & 0.001 & -0.092 & -2.763 & 0.006 \\
\hline \multirow[t]{3}{*}{ ApoAI/ApoB } & Waist circumference & -0.020 & 0.002 & -0.212 & -8.461 & 0.000 \\
\hline & Age & -0.005 & 0.001 & -0.102 & -4.101 & 0.000 \\
\hline & Alcohol consumption & 0.001 & 0.001 & 0.049 & 1.975 & 0.048 \\
\hline
\end{tabular}

B, Han

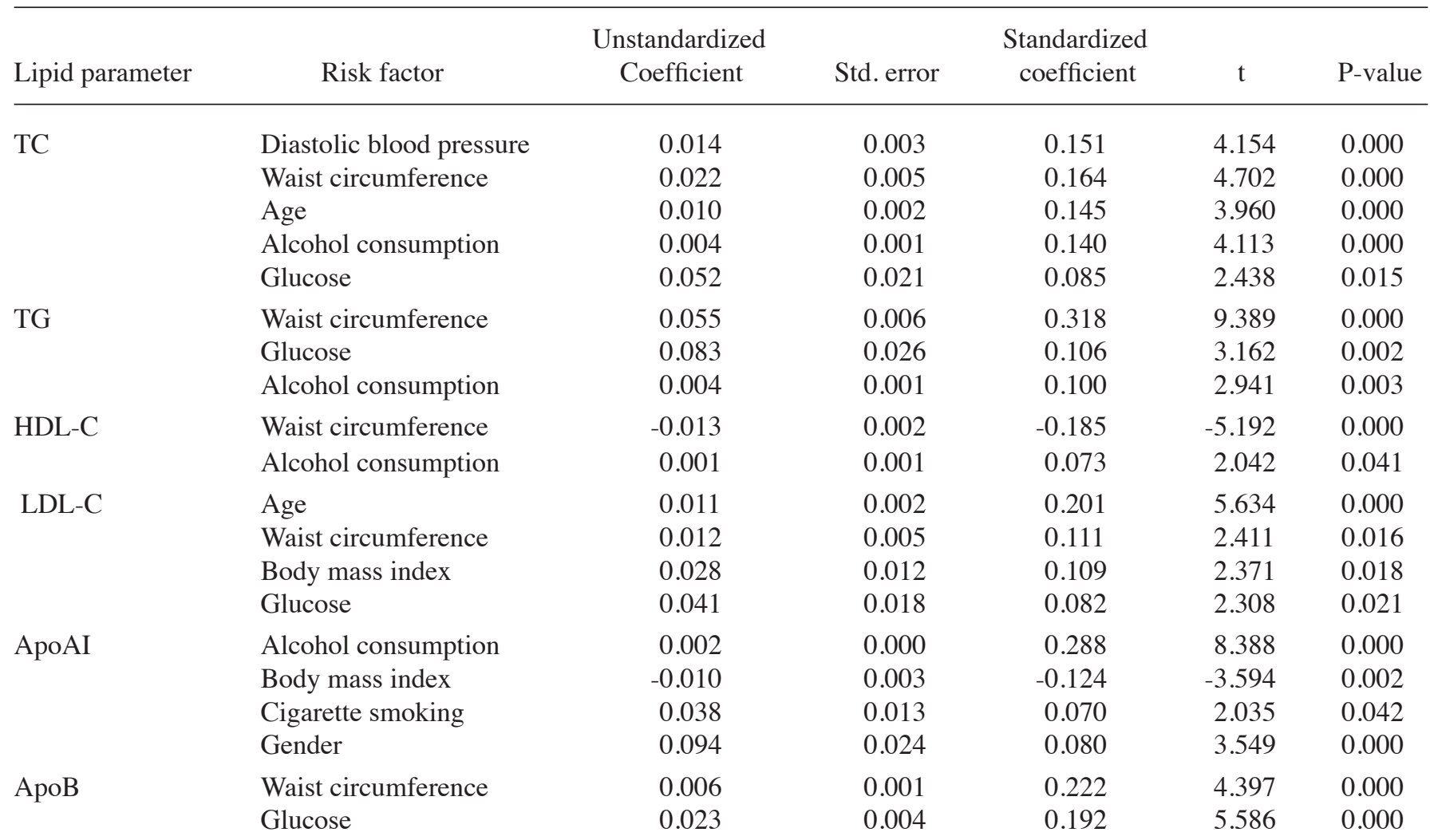


Table IV. Continued.

\begin{tabular}{|c|c|c|c|c|c|c|}
\hline Lipid parameter & Risk factor & $\begin{array}{l}\text { Unstandardized } \\
\text { Coefficient }\end{array}$ & Std. error & $\begin{array}{c}\text { Standardized } \\
\text { coefficient }\end{array}$ & $\mathrm{t}$ & P-value \\
\hline \multirow{8}{*}{ ApoAI/ApoB } & Alcohol consumption & 0.048 & 0.011 & 0.152 & 4.430 & 0.000 \\
\hline & Systolic blood pressure & 0.001 & 0.000 & 0.133 & 3.823 & 0.000 \\
\hline & Body mass index & 0.010 & 0.003 & 0.143 & 2.837 & 0.005 \\
\hline & Waist circumference & -0.013 & 0.003 & -0.198 & -4.352 & 0.000 \\
\hline & Gender & -0.006 & 0.001 & -0.099 & -2.824 & 0.005 \\
\hline & Body mass index & -0.015 & 0.007 & -0.171 & -3.780 & 0.000 \\
\hline & Glucose & -0.026 & 0.010 & -0.090 & -2.588 & 0.010 \\
\hline & Alcohol consumption & 0.001 & 0.001 & 0.068 & 2.011 & 0.045 \\
\hline \multicolumn{7}{|l|}{ C, Mulao } \\
\hline Lipid parameter & Risk factor & $\begin{array}{l}\text { Unstandardized } \\
\text { Coefficient }\end{array}$ & Std. error & $\begin{array}{l}\text { Standardized } \\
\text { coefficient }\end{array}$ & $\mathrm{t}$ & P-value \\
\hline \multirow[t]{2}{*}{$\mathrm{TC}$} & Age & 0.015 & 0.003 & 0.181 & 5.083 & 0.000 \\
\hline & Alcohol consumption & 0.002 & 0.001 & 0.074 & 2.087 & 0.037 \\
\hline \multirow[t]{2}{*}{ TG } & Waist circumference & 0.030 & 0.004 & 0.268 & 7.514 & 0.000 \\
\hline & Systolic blood pressure & 0.004 & 0.002 & 0.090 & 2.521 & 0.012 \\
\hline \multirow[t]{4}{*}{ HDL-C } & Body mass index & -0.023 & 0.008 & -0.156 & -3.018 & 0.003 \\
\hline & Alcohol consumption & 0.001 & 0.000 & 0.114 & 3.277 & 0.001 \\
\hline & Waist circumference & -0.009 & 0.003 & -0.171 & -3.276 & 0.001 \\
\hline & Age & 0.002 & 0.001 & 0.076 & 2.194 & 0.029 \\
\hline \multirow[t]{2}{*}{ LDL-C } & Gender & 0.341 & 0.065 & 0.183 & 5.219 & 0.000 \\
\hline & Body mass index & 0.055 & 0.010 & 0.182 & 5.203 & 0.000 \\
\hline \multirow[t]{3}{*}{ ApoAI } & Alcohol consumption & 0.002 & 0.000 & 0.157 & 4.388 & 0.000 \\
\hline & Waist circumference & -0.009 & 0.003 & -0.190 & -3.535 & 0.000 \\
\hline & Body mass index & 0.015 & 0.007 & 0.113 & 2.188 & 0.034 \\
\hline \multirow[t]{3}{*}{ ApoB } & Waist circumference & 0.012 & 0.001 & 0.188 & 5.121 & 0.000 \\
\hline & Systolic blood pressure & 0.005 & 0.002 & 0.184 & 3.851 & 0.000 \\
\hline & Diastolic blood pressure & -0.018 & 0.001 & -0.162 & -3.367 & 0.001 \\
\hline \multirow[t]{3}{*}{ ApoAI/ApoB } & Waist circumference & -0.020 & 0.004 & -0.183 & -4.955 & 0.000 \\
\hline & Age & -0.008 & 0.002 & -0.131 & -3.572 & 0.000 \\
\hline & Diastolic blood pressure & 0.006 & 0.003 & 0.077 & 2.038 & 0.042 \\
\hline
\end{tabular}

TC, total cholesterol; TG, triglyceride; HDL-C, high-density lipoprotein cholesterol; LDL-C, low-density lipoprotein cholesterol; ApoAI, apolipoprotein AI; ApoB, apolipoprotein B.

Han populations ( $\mathrm{P}<0.01$ for each). Significant differences were identified in the genotypic and allelic frequencies between males and females in the Mulao population $(\mathrm{P}<0.01)$, however, not in the Han population.

Genotypes and serum lipid levels. As shown in Table III, the levels of TG in the Mulao population, but not in the Han population, were different among the three genotypes $(\mathrm{P}<0.05)$, the A allele carriers exhibited lower TG levels than the A allele noncarriers.

Risk factors for serum lipid parameters. As shown in Tables IV and $\mathrm{V}$, serum lipid parameters were also correlated with several environmental factors, including age, gender, alcohol consumption, cigarette smoking, blood pressure, blood glucose levels and BMI in the two ethnic groups $(\mathrm{P}<0.05-0.001)$.

\section{Discussion}

The present study demonstrated that serum LDL-C and ApoB levels were higher in the Mulao than in the Han population. No significant differences in the levels of TC, TG, HDL-C, ApoAI and the ratio of ApoAI to ApoB between the two ethnic groups were identified. It is well known that dyslipidemia is a complex trait caused by environmental and genetic factors. Family and twin studies suggest that in numerous populations, $\sim 40-60 \%$ of the variation in serum lipid profiles is genetically determined (7-11). 
Table V.Association between serum lipid parameters and relative factors in males and females of the Mulao and Han populations.

A, Han/male

\begin{tabular}{|c|c|c|c|c|c|c|}
\hline Lipid parameter & Risk factor & $\begin{array}{l}\text { Unstandardized } \\
\text { Coefficient }\end{array}$ & Std. error & $\begin{array}{c}\text { Standardized } \\
\text { coefficient }\end{array}$ & $\mathrm{t}$ & P-value \\
\hline \multirow[t]{3}{*}{$\mathrm{TC}$} & Age & 0.014 & 0.004 & 0.213 & 3.677 & 0.000 \\
\hline & Waist circumference & 0.021 & 0.008 & 0.164 & 2.824 & 0.005 \\
\hline & Diastolic blood pressure & 0.013 & 0.005 & 0.143 & 2.385 & 0.018 \\
\hline \multirow[t]{2}{*}{ TG } & Waist circumference & 0.049 & 0.007 & 0.361 & 6.557 & 0.000 \\
\hline & Alcohol consumption & 0.004 & 0.002 & 0.114 & 2.073 & 0.039 \\
\hline HDL-C & Waist circumference & -0.012 & 0.003 & -0.222 & -3.838 & 0.000 \\
\hline \multirow[t]{3}{*}{ LDL-C } & Age & 0.013 & 0.003 & 0.239 & 4.064 & 0.000 \\
\hline & Body mass index & 0.044 & 0.016 & 0.158 & 2.783 & 0.006 \\
\hline & Glucose & 0.058 & 0.028 & 0.121 & 2.048 & 0.041 \\
\hline ApoAI & Alcohol consumption & 0.002 & 0.000 & 0.241 & 4.158 & 0.000 \\
\hline \multirow[t]{3}{*}{ Apo B } & Waist circumference & 0.008 & 0.001 & 0.294 & 5.283 & 0.000 \\
\hline & Diastolic blood pressure & 0.001 & 0.001 & 0.133 & 2.161 & 0.032 \\
\hline & Glucose & 0.013 & 0.006 & 0.116 & 2.040 & 0.042 \\
\hline \multirow[t]{2}{*}{ ApoAI/ApoB } & Waist circumference & -0.016 & 0.004 & -0.249 & -4.356 & 0.000 \\
\hline & Age & -0.004 & 0.002 & -0.124 & -2.165 & 0.031 \\
\hline
\end{tabular}

B, Han/female

\begin{tabular}{|c|c|c|c|c|c|c|}
\hline Lipid parameter & Risk factor & $\begin{array}{l}\text { Unstandardized } \\
\text { Coefficient }\end{array}$ & Std. error & $\begin{array}{c}\text { Standardized } \\
\text { coefficient }\end{array}$ & $\mathrm{t}$ & P-value \\
\hline \multirow[t]{4}{*}{$\mathrm{TC}$} & Diastolic blood pressure & 0.014 & 0.004 & 0.146 & 3.180 & 0.002 \\
\hline & Waist circumference & 0.024 & 0.006 & 0.177 & 4.052 & 0.000 \\
\hline & Alcohol consumption & 0.006 & 0.001 & 0.187 & 4.350 & 0.000 \\
\hline & Age & 0.010 & 0.003 & 0.145 & 3.265 & 0.001 \\
\hline \multirow[t]{3}{*}{ TG } & Waist circumference & 0.058 & 0.008 & 0.302 & 7.016 & 0.000 \\
\hline & Glucose & 0.115 & 0.032 & 0.128 & 3.016 & 0.003 \\
\hline & Alcohol consumption & 0.004 & 0.002 & 0.092 & 2.157 & 0.032 \\
\hline HDL-C & Waist circumference & -0.012 & 0.003 & -0.158 & -3.532 & 0.000 \\
\hline \multirow[t]{2}{*}{ LDL-C } & Waist circumference & 0.024 & 0.005 & 0.217 & 4.977 & 0.000 \\
\hline & Age & 0.010 & 0.002 & 0.185 & 4.248 & 0.000 \\
\hline \multirow[t]{2}{*}{ ApoAI } & Alcohol consumption & 0.002 & 0.062 & 0.307 & 7.120 & 0.000 \\
\hline & Body mass index & -0.010 & 0.000 & -0.130 & -3.017 & 0.003 \\
\hline \multirow[t]{4}{*}{ ApoB } & Waist circumference & 0.020 & 0.003 & 0.312 & 7.302 & 0.000 \\
\hline & Systolic blood pressure & 0.021 & 0.005 & 0.178 & 4.011 & 0.000 \\
\hline & Alcohol consumption & 0.003 & 0.001 & 0.258 & 5.633 & 0.000 \\
\hline & Glucose & -0.150 & 0.047 & -0.142 & -3.208 & 0.000 \\
\hline \multirow[t]{4}{*}{ ApoAI/ApoB } & Waist circumference & -0.014 & 0.003 & -0.228 & -4.168 & 0.000 \\
\hline & Body mass index & -0.024 & 0.008 & -0.168 & -3.099 & 0.002 \\
\hline & Glucose & -0.028 & 0.013 & -0.095 & -2.176 & 0.030 \\
\hline & Age & -0.003 & 0.001 & -0.093 & -2.137 & 0.033 \\
\hline
\end{tabular}

C, Mulao/male

\begin{tabular}{llcrrrr}
\hline Lipid parameter & \multicolumn{1}{c}{ Risk factor } & $\begin{array}{c}\text { Unstandardized } \\
\text { Coefficient }\end{array}$ & Std. error & $\begin{array}{c}\text { Standardized } \\
\text { coefficient }\end{array}$ & P-value \\
\hline TC & Age & 0.036 & 0.010 & 0.195 & 3.422 & 0.001 \\
& Waist circumference & 0.020 & 0.010 & 0.115 & 2.019 & 0.044 \\
TG & Waist circumference & 0.036 & 0.006 & 0.316 & 5.842 & 0.000 \\
& Alcohol consumption & 0.010 & 0.001 & 0.123 & 2.267 & 0.024 \\
HDL-C & Waist circumference & -0.018 & 0.003 & -0.299 & -5.296 & 0.000
\end{tabular}


Table V. Continued.

\begin{tabular}{llccccc}
\hline Lipid parameter & \multicolumn{1}{c}{ Risk factor } & $\begin{array}{c}\text { Unstandardized } \\
\text { Coefficient }\end{array}$ & Std. error & $\begin{array}{c}\text { Standardized } \\
\text { coefficient }\end{array}$ & $\mathrm{t}$ & P-value \\
\hline HDL-C & Waist circumference & -0.018 & 0.003 & -0.299 & -5.296 & 0.000 \\
& Age & 0.007 & 0.004 & 0.114 & 2.030 & 0.043 \\
LDL-C & Body mass index & 0.051 & 0.016 & 0.181 & 3.149 & 0.002 \\
& Age & 0.016 & 0.007 & 0.135 & 2.351 & 0.019 \\
Apo AI & Age & 0.010 & 0.003 & 0.185 & 3.310 & 0.001 \\
& Alcohol consumption & 0.002 & 0.001 & 0.146 & 2.606 & 0.030 \\
ApoB & Waist circumference & 0.019 & 0.004 & 0.246 & 4.435 & 0.000 \\
ApoAI/ApoB & Waist circumference & -0.020 & 0.009 & -0.123 & -2.167 & 0.031 \\
\hline
\end{tabular}

$\mathrm{D}$, Mulao/female

\begin{tabular}{|c|c|c|c|c|c|c|}
\hline Lipid parameter & Risk factor & $\begin{array}{c}\text { Unstandardized } \\
\text { Coefficient }\end{array}$ & Std. error & $\begin{array}{l}\text { Standardized } \\
\text { coefficient }\end{array}$ & $\mathrm{t}$ & P-value \\
\hline $\mathrm{TC}$ & Age & 0.019 & 0.005 & 0.192 & 3.849 & 0.000 \\
\hline \multirow[t]{2}{*}{ TG } & Waist circumference & 0.025 & 0.014 & 0.231 & 4.975 & 0.000 \\
\hline & Systolic blood pressure & 0.005 & 0.005 & 0.114 & 2.451 & 0.015 \\
\hline \multirow[t]{2}{*}{ HDL-C } & Body mass index & -0.043 & 0.006 & -0.302 & -6.758 & 0.000 \\
\hline & Alcohol consumption & 0.001 & 0.000 & 0.12 & 2.796 & 0.005 \\
\hline LDL-C & Body mass index & 0.050 & 0.014 & 0.164 & 3.530 & 0.000 \\
\hline \multirow[t]{2}{*}{ ApoAI } & Waist circumference & -0.007 & 0.002 & -0.160 & -3.459 & 0.001 \\
\hline & Alcohol consumption & 0.001 & 0.001 & 0.141 & 3.065 & 0.002 \\
\hline \multirow[t]{2}{*}{ ApoB } & Waist circumference & 0.008 & 0.003 & 0.143 & 3.075 & 0.002 \\
\hline & Cigarette smoking & 1.197 & 0.340 & 0.172 & 3.526 & 0.000 \\
\hline \multirow[t]{3}{*}{ ApoAI/ApoB } & Waist circumference & -0.020 & 0.003 & -0.286 & -6.159 & 0.000 \\
\hline & Age & -0.009 & 0.003 & -0.133 & -2.935 & 0.004 \\
\hline & Diastolic blood pressure & 0.006 & 0.002 & 0.109 & 2.316 & 0.021 \\
\hline
\end{tabular}

TC, total cholesterol; TG, triglyceride; HDL-C, high-density lipoprotein cholesterol; LDL-C, low-density lipoprotein cholesterol; ApoAI, apolipoprotein AI; ApoB, apolipoprotein B.

Mulao is a relatively conservative minority. Lack of communication with the other ethnic groups, their original life habits and culture are still completely conserved to the present day. Glutinous rice and salted foods are their staple diet. Marriages arranged by parents were common. Prenatal betrothal and cousin marriages were also popular in this minority. Brides did not live with their husbands until the first child was born (38). Therefore, it is considered that the hereditary characteristics and genotypes of certain lipid metabolism-related genes in this population may be different from those in the Han Chinese.

The genotypic and allelic frequencies of the rs7134375 SNP in diverse racial/ethnic groups are different, which can be found on the International HapMap project website (http://hapmap.ncbi. nlm.nih.gov/cgi-perl/gbrowse/hapmap24_B36/\#search). The frequency of CC, CA and AA genotypes was 36.7, 43.3 and $20.0 \%$ in Utah residents with ancestry from Northern and Western Europe (CEU); 47.5, 42.4 and 10.2\% in Yoruba in Ibadan, Nigeria (YRI); 54.5, 45.5 and $0 \%$ in Japanese in Tokyo, Japan (JPT) and 53.3, 42.2 and 4.4\% in Han Chinese in Beijing, China (CHB). The frequency of $\mathrm{C}$ and $\mathrm{A}$ alleles was 58.3 and
41.7\% in CEU; 68.6 and $31.4 \%$ in YRI; 77.3 and $22.7 \%$ in JPT and 74.4 and $25.6 \%$ in CHB. The present study identified significant differences in the genotypic frequency of the $P D E 3 A$ rs7134375 SNP between the two ethnic groups. The frequency of the CC genotype was lower in the Mulao than in the Han population and the frequency of the $\mathrm{C}$ allele was lower in the Mulao than in the Han population $(72.14$ vs. $78.55 \%$; $\mathrm{P}<0.001)$. These results suggest that the prevalence of the rs7134375 SNP in PDE3A may exhibit a racial/ethnic difference.

The potential association between the rs7134375 SNP and plasma or serum lipid levels in humans has been evaluated in several previous GWAS. The rs7134375 SNP has been associated with serum HDL-C levels (12). At present, no studies have verified that it is associated with other lipid parameters. The current study demonstrated that the A allele carriers in the Mulao population; however, not in the Han population, were associated with lower serum TG levels than the A allele noncarriers. These results suggest that the associations of the rs7134375 SNP in PDE3A and serum lipid phenotypes may have a racial/ethnic specificity. cAMP and cGMP are important second messengers involved in intracellular signal transduction 
in numerous cell types, including vascular smooth muscle cells (VSMCs) (39). The biological processes regulated by cAMP and cGMP in VSMCs include cell contractility, proliferation, migration, apoptosis and inflammatory responses, all of which have been implicated in the regulation of atherogenesis and post angioplasty restenosis (40). Cyclic nucleotide PDEs are important in regulating intracellular cyclic nucleotide (cAMP and cGMP) levels and compartmentalization via degradation of cyclic nucleotides. PDE3 is the major cAMP-hydrolyzing PDE present in VSMCs and oocytes, and its inhibition by nitric oxide-induced accumulation of cGMP results in increased cAMP and protein kinase A activity (41-43). Begum et al (44) revealed that therapies specifically aimed at inhibiting the PDE3A isoform may decrease atherosclerosis, and improve metabolic syndrome and post angioplasty restenosis. No clear mechanism on how PDE3 affects the levels of blood lipids was identified; however, it is hypothesized that PDE3 affects blood lipid levels by interfering with the expression of cAMP and cGMP.

Furthermore, exposure to different lifestyles and environments in the populations resident in Guangxi may further modify the effects of genetic variation on blood lipids. The present study demonstrated that serum lipid parameters were correlated with age, gender, alcohol consumption, cigarette smoking, BMI and blood pressure in the two ethnic groups. These data suggest that environmental factors are also important in determining serum lipid levels in populations. Although Mulao and Han nationalities reside in the same region, the diet and lifestyles are different between the two ethnic groups. The individuals of the Mulao nationality are more inclined to eat cold foods along with acidic and spicy dishes. They also enjoy eating animal offal, which contains an abundance of saturated fatty acids. On the contrary, the Han people prefer to eat relatively healthy diets, which include high-quality protein, low-saturated fatty acids, and vitamin-rich vegetables and fruits. The effects of dietary macronutrients on serum lipid levels and their effects on CHD have been extensively studied $(45,46)$. Serum cholesterol concentrations in humans respond more to the major dietary nutrients than to dietary cholesterol itself. Higher serum TC and TG levels have been found in the populations consuming high-saturated fatty acid diets, whereas lower levels of them have been noted in the populations with high carbohydrate or monounsaturated fatty acid diets. Thus, the findings of the present study may be helpful in identifying susceptibility genes, changing unhealthy lifestyles, and preventing environmental risk factors for lipid-related diseases.

In conclusion, the genotypic and allelic frequencies of the rs7134375 SNP were different between the Mulao and Han populations. The associations of the SNP and serum lipid levels were also different between the two ethnic groups. The PDE3A rs7134375 SNP was shown to be associated with serum TG levels in the Mulao, however, not in the Han populations. In addition, the A allele carriers were shown to exhibit lower TG levels than the A allele noncarriers.

\section{Acknowledgements}

This study was supported by the National Natural Science Foundation of China (grant no. 30960130) and the Guangxi Natural Science Foundation (grant no.2012GXNSFAA276011).

\section{References}

1. Lopez AD, Mathers CD, Ezzati M, Jamison DT and Murray CJ: Global and regional burden of disease and risk factors, 2001: systematic analysis of population health data. Lancet 367 : 1747-1757, 2006.

2. Ezzati M,Lopez AD, Rodgers A, Vander Hoorn S and Murray CJ: Comparative Risk Assessment Collaborating Group. Selected major risk factors and global and regional burden of disease. Lancet 360: 1347-1360, 2002.

3. Law MR, Wald NJ and Rudnicka AR: Quantifying effect of statins on low density lipoprotein cholesterol, ischaemic heart disease, and stroke: systematic review and meta-analysis. BMJ 326: 1423, 2003.

4. Kuulasmaa K, Tunstall-Pedoe H, Dobson A, et al: Estimation of contribution of changes in classic risk factors to trends in coronary-event rates across the WHO MONICA Project populations. Lancet 355: 675-687, 2000.

5. Clarke R, Emberson JR, Parish S, et al: Cholesterol fractions and apolipoproteins as risk factors for heart disease mortality in older men. Arch Intern Med 167: 1373-1378, 2007.

6. Qian DF, Fan GL, Chen P, et al: Risk factors for hyperuricemia in active and retired employees underwent physical examination. Zhonghua Xin Xue Guan Bing Za Zhi 41: 60-64, 2013 (In Chinese).

7. Ruixing Y, Yuming C, Shangling P, et al: Effects of demographic, dietary and other lifestyle factors on the prevalence of hyperlipidemia in Guangxi Hei Yi Zhuang and Han populations. Eur J Cardiovasc Prev Rehabil 13: 977-984, 2006.

8. Ruixing Y, Qiming F, Dezhai Y, et al: Comparison of demography, diet, lifestyle, and serum lipid levels between the Guangxi Bai Ku Yao and Han populations. J Lipid Res 48: 2673-2681, 2007.

9. Ruixing Y, Yiyang L, Meng L, et al: Interactions of the apolipoprotein $\mathrm{C}$-III 3238C $>$ G polymorphism and alcohol consumption on serum triglyceride levels. Lipids Health Dis 9: 86, 2010.

10. Yin RX, Li YY, Liu WY, Zhang L and Wu JZ: Interactions of the apolipoprotein A5 gene polymorphisms and alcohol consumption on serum lipid levels. PLoS One 6: e17954, 2011.

11. Pilia G, Chen WM, Scuteri A, et al: Heritability of cardiovascular and personality traits in 6,148 Sardinians. PLoS Genet 2: e132, 2006.

12. Teslovich TM, Musunuru K, Smith AV, et al: Biological, clinical and population relevance of 95 loci for blood lipids. Nature 466: 707-713, 2010.

13. Kathiresan S, Melander O, Guiducci C, et al: Six new loci associated with blood low-density lipoprotein cholesterol, high-density lipoprotein cholesterol or triglycerides in humans. Nat Genet 40: 189-197, 2008.

14. Sandhu MS, Waterworth DM, Debenham SL, et al: LDL-cholesterol concentrations: a genome-wide association study. Lancet 371: 483-491, 2008.

15. Willer CJ, Sanna S, Jackson AU, et al: Newly identified loci that influence lipid concentrations and risk of coronary artery disease. Nat Genet 40: 161-169, 2008.

16. Wallace C, Newhouse SJ, Braund P, et al: Genome-wide association study identifies genes for biomarkers of cardiovascular disease: serum urate and dyslipidemia. Am J Hum Genet 82: 139-149, 2008.

17. López S, Buil A, Ordoñez J, et al: Genome-wide linkage analysis for identifying quantitative trait loci involved in the regulation of lipoprotein a (Lpa) levels. Eur J Hum Genet 16: 1372-1379, 2008.

18. Aulchenko YS, Ripatti S, Lindqvist I, et al: Loci influencing lipid levels and coronary heart disease risk in 16 European population cohorts. Nat Genet 41: 47-55, 2009.

19. Li X, Monda KL, Göring HH, et al: Genome-wide linkage scan for plasma high density lipoprotein cholesterol, apolipoprotein A-1 and triglyceride variation among American Indian populations: the Strong Heart Family Study. J Med Genet 46: 472-479, 2009

20. Chasman DI, Paré G, Zee RY, et al: Genetic loci associated with plasma concentration of low-density lipoprotein cholesterol, high-density lipoprotein cholesterol, triglycerides, apolipoprotein A1, and Apolipoprotein B among 6382 white women in genome-wide analysis with replication. Circ Cardiovasc Genet 1: 21-30, 2008.

21. Waterworth DM, Ricketts SL, Song K, et al: Genetic variants influencing circulating lipid levels and risk of coronary artery disease. Arterioscler Thromb Vasc Biol 30: 2264-2276, 2010.

22. Zhang Z, Tao L, Chen Z, Zhou D, Kan M, Zhang D, Li C, He L and Liu Y: Association of genetic loci with blood lipids in the Chinese population. PLoS One 6: e27305, 2011. 
23. Dumitrescu L, Carty CL, Taylor K, et al: Genetic determinants of lipid traits in diverse populations from the population architecture using genomics and epidemiology (PAGE) study. PLoS Genet 7: e1002138, 2011.

24. Omori K and Kotera J: Overview of PDEs and their regulation. Circ Res 100: 309-327, 2007

25. Bender AT and Beavo JA: Cyclic nucleotide phosphodiesterases: molecular regulation to clinical use. Pharmacol Rev 58: 488-520, 2006.

26. Thompson PE, Manganiello V and Degerman E: Re-discovering PDE3 inhibitors - new opportunities for a long neglected target. Curr Top Med Chem 7: 421-436, 2007.

27. Begum N,Hockman S and Manganiello VC: Phosphodiesterase $3 \mathrm{~A}$ (PDE3A) deletion suppresses proliferation of cultured murine vascular smooth muscle cells (VSMCs) via inhibition of mitogen-activated protein kinase (MAPK) signaling and alterations in critical cell cycle regulatory proteins. J Biol Chem 286 26238-26249, 2011.

28. Sun B, Li H, Shakur Y, et al: Role of phosphodiesterase type $3 \mathrm{~A}$ and $3 \mathrm{~B}$ in regulating platelet and cardiac function using subtype-selective knockout mice. Cell Signal 19: 1765-1771, 2007.

29. Jeemon P, Pettigrew K, Sainsbury C, Prabhakaran D and Padmanabhan S: Implications of discoveries from genome-wide association studies in current cardiovascular practice. World J Cardiol 3: 230-247, 2011.

30. National Bureau of Statistics of the People's Republic of China: Population distribution of 2000 census in China. http://www. stats.gov.cn/tjsj/ndsj/renkoupucha/2000 pucha/html/t0201.htm.

31. Xu L, Deng QY, Li SF, Zhou LN, Gong JC and Wei BY: Genetic analysis of Mulao nationality using 15 short tandem repeats. Zhonghua Yi Xue Yi Chuan Xue Za Zhi 25: 96-100, 2008 (In Chinese)

32. Li Q, Yin RX, Yan TT, et al: Association of the GALNT2 gene polymorphisms and several environmental factors with serum lipid levels in the Mulao and Han populations. Lipids Health Dis 10: 160, 2011.

33. Yan TT, Yin RX, Li Q, et al: Sex-specific association of rs16996148 SNP in the NCAN/CILP2/PBX4 and serum lipid levels in the Mulao and Han populations. Lipids Health Dis 10: 248, 2011.

34. People's Republic of China - United States Cardiovascular and Cardiopulmonary Epidemiology Research Group: An epidemiological study of cardiovascular and cardiopulmonary disease risk factors in four populations in the People's Republic of China. Baseline report from the P.R.C.-U.S.A. Collaborative Study. Circulation 85: 1083-1096, 1992.
35. Wu DF, Yin RX, Hu XJ, et al: Association of rs5888 SNP in the scavenger receptor class B type 1 gene and serum lipid levels. Lipids Health Dis 11: 50, 2012.

36. Ruixing Y, Weixiong L, Hanjun Y, et al: Diet, lifestyle, and blood pressure of the middle-aged and elderly in the Guangxi Bai Ku Yao and Han populations. Am J Hypertens 21: 382-387, 2008.

37. Zhou BF: Effect of body mass index on all-cause mortality and incidence of cardiovascular diseases - report for meta-analysis of prospective studies open optimal cut-off points of body mass index in Chinese adults. Biomed Environ Sci 15: 245-252, 2002.

38. Hongkong SHS Group Company Limited: The Mulao ethnic minority. http://arts.imextrade.ru/education/nationalities-ofchina/21-china-mulaozu/?lang=en.

39. Lincoln TM, Wu X, Sellak H, Dey N and Choi CS: Regulation of vascular smooth muscle cell phenotype by cyclic GMP and cyclic GMP-dependent protein kinase. Front Biosci 11: 356-367, 2006.

40. Ross R: The pathogenesis of atherosclerosis: a perspective for the 1990s. Nature 362: 801-809, 1993.

41. Francis SH, Busch JL, Corbin JD and Sibley D: cGMP-dependent protein kinases and cGMP phosphodiesterases in nitric oxide and cGMP action. Pharmacol Rev 62 $525-563,2010$

42. Aizawa T, Wei H, Miano JM, Abe J, Berk BC and Yan C: Role of phosphodiesterase 3 in NO/cGMP-mediated antiinflammatory effects in vascular smooth muscle cells. Circ Res 93: 406-413, 2003.

43. Kass DA, Takimoto E, Nagayama $T$ and Champion HC: Phosphodiesterase regulation of nitric oxide signaling. Cardiovasc Res 75: 303-314, 2007.

44. Begum N, Shen W and Manganiello V: Role of PDE3A in regulation of cell cycle progression in mouse vascular smooth muscle cells and oocytes: implications in cardiovascular diseases and infertility. Curr Opin Pharmacol 11: 725-729, 2011.

45. Bermudez OI, Velez-Carrasco W, Schaefer EJ and Tucker KL: Dietary and plasma lipid, lipoprotein, and apolipoprotein profiles among elderly Hispanics and non-Hispanics and their association with diabetes. Am J Clin Nutr 76: 1214-1221, 2002.

46. Liu WY, Yin RX, Zhang L, Wu DF, Htet Aung LH, Hu XJ, Cao XL and Miao L: Interactions of the LIPG 584C $>$ T polymorphism and alcohol consumption on serum lipid levels. Alcohol 45: 681-687, 2011. 